\title{
The Molecular Basis of Rectal Cancer
}

\author{
Michelle Shiller, DO ${ }^{1}$ Sarah Boostrom, MD² \\ ${ }^{1}$ Department of Pathology, Baylor University Medical Center at Dallas, \\ Dallas, Texas \\ 2 Department of Surgery, Baylor University Medical Center at Dallas, \\ Dallas, Texas
}

Address for correspondence Michelle Shiller, DO, Department of Pathology, Baylor University Medical Center at Dallas, 3500 Gaston Avenue, Dallas, TX 75246 (e-mail: Shirley.Shiller@baylorhealth.edu).

Clin Colon Rectal Surg 2015;28:53-60.
Abstract
Keywords
- colorectal cancer
- molecular testing
- hereditary cancer
- adenoma-carcinoma sequence

The majority of rectal carcinomas are sporadic in nature, and relevant testing for driver mutations to guide therapy is important. A thorough family history is necessary and helpful in elucidating a potential hereditary predilection for a patient's carcinoma. The adequate diagnosis of a heritable tendency toward colorectal carcinoma alters the management of a patient disease and permits the implementation of various surveillance algorithms as preventive measures.

\section{Sporadic Colorectal Cancer}

The overwhelming majority of colorectal cancer is sporadic and evolves by three main pathways: the adenoma-carcinoma sequence, $\mathrm{CpG}$ island hypermethylation, or mismatch repair dysfunction. Regardless of the process, multiple mutations must be acquired, with epigenetic events occurring to ultimately lead to carcinogenesis. Universally, chromosomal instability is the most common pathway to colorectal adenocarcinoma. ${ }^{1,2}$

WNT signaling is the classic pathway under which most rectal cancers evolve, with an initial injury to the $A P C$ gene (5q21) which may be present in the germline (inherited) or somatic (acquired). In the setting of sporadic colorectal cancer, the initial mutation is acquired. Ultimately, a second $A P C$ mutation is acquired, which decreases the downregulation of $\beta$-catenin. The result of unregulated $\beta$-catenin activity is translocation of $\beta$-catenin to the nucleus, upregulating transcription of MYC, and CyclinD1, both of which are proliferative factors. With these changes, the mucosa now has a milieu predisposed to neoplasia, and additional genetic events further perpetuate the sequence such as acquisition of activating mutations in oncogenes such as KRAS (codons 12, 13, and 61), BRAF (V600E $>>>V 600 \mathrm{~K}>\mathrm{V} 600 \mathrm{H})$, PIK3CA, and others (- Fig. 1). Accumulation of such genetic aberrations is a late event; less than $10 \%$ of adenomas smaller than $1 \mathrm{~cm}$ in size contain activating KRAS mutations. However, more than $50 \%$ of adenomas greater than $1 \mathrm{~cm}$ in size possess
KRAS activating mutations. Additional events in the progression toward carcinoma include inactivating tumor suppressor genes such as SMAD4, and SMAD2, which are part of the TGF $\beta$ signaling pathway, conferring autonomous growth. Deleted in colorectal cancer $(D C C)$, also a tumor suppressor gene, and proximal to SMAD4 and SMAD2 is another gene involved in the acquisition of genetic events resulting in invasive carcinoma. It is no surprise that p53, the "guardian of the genome," and the most common denominator for neoplasia, is also part of the progression toward invasive colorectal adenocarcinoma. Moreover, acquisition of a p53 mutation is also noted in invasive lesions, but in few adenomas, implicating that this is a late event in the carcinoma progression. ${ }^{1,2}$

Though less common, sporadic mismatch repair deficiency is another pathway toward development of colorectal carcinoma. This is due to hypermethylation of the promoter region of MLH1. MLH1 and PMS2 comprise a heterodimer that monitors DNA base pairing (MMR). When hypermethylated, expression of this protein is silenced and therefore not expressed in protein immunohistochemistry (IHC) studies. Microsatellite instability (MSI) studies, when performed in these cases, will be positive, due to slippage of the DNA mismatch repair mechanism. It is important to have a thorough family history as histomorphology alone does not distinguish sporadic colorectal carcinoma from its inherited counterpart, Lynch syndrome. Although a family history alone is not the only solution to this possibility, it can be very instrumental in driving the correct diagnostic algorithm
Issue Theme Rectal Cancer;

Guest Editors: James W. Fleshman, MD, FACS, FASCRS, and Warren E. Lichliter, MD, FACS, FASCRS
Copyright (c) 2015 by Thieme Medical Publishers, Inc., 333 Seventh Avenue, New York, NY 10001, USA. Tel: +1(212) 584-4662.
Dol http://dx.doi.org/ 10.1055/s-0035-1545070. ISSN 1531-0043. 


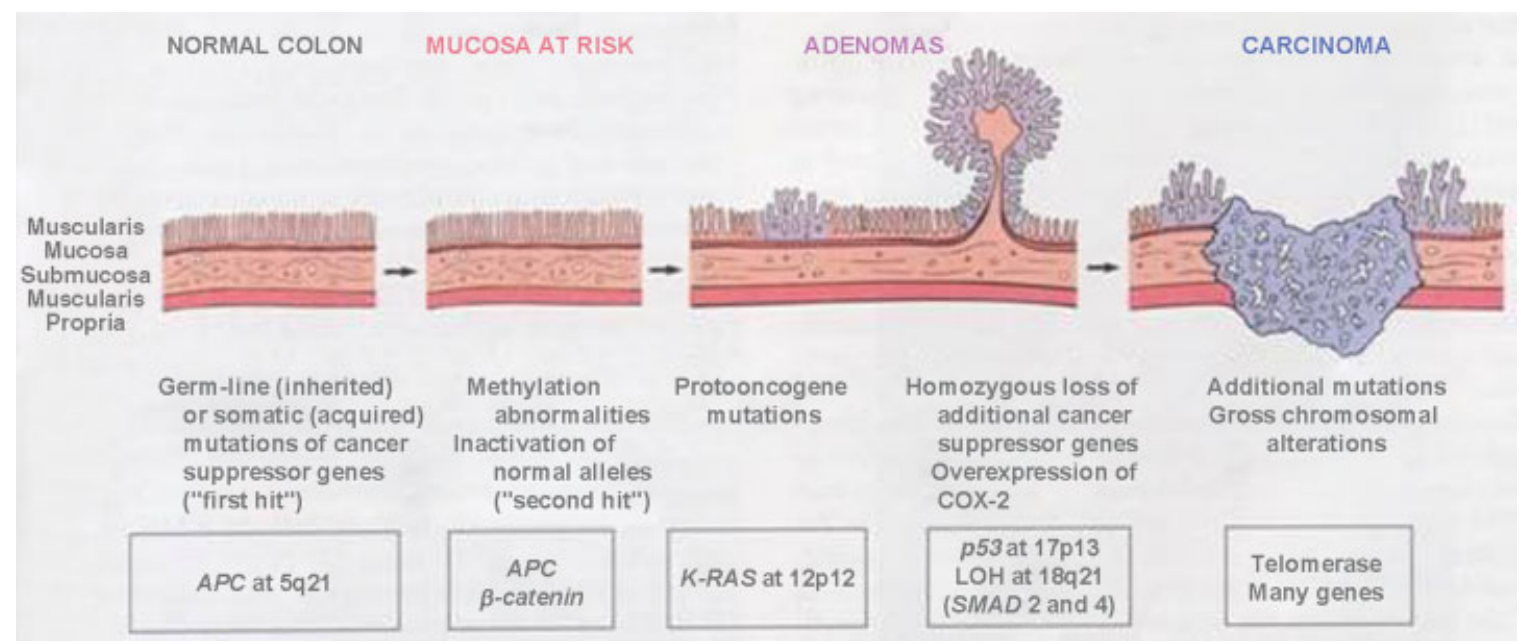

Fig. 1 Sequence of mutations.

and therefore offering the patient the best follow-up care regarding their cancer diagnosis, especially since, like their hereditary counterparts, these lesions may reside in the right colon and may have mucinous differentiation and tumor infiltrating lymphocytes. Often, BRAF mutations are present in these cancers, in which testing can confirm their sporadic nature and rule out the possibility of a hereditary predisposition to colorectal carcinoma. Finally, KRAS activating mutations and inactivation of $p 53$ are not commonly associated with colorectal carcinoma due to sporadic loss of DNA mismatch repair. ${ }^{1,2}$

A third pathway to sporadic colorectal carcinoma, and the least common of all, is CpG island hypermethylation. BRAF mutations are associated with $\mathrm{CpG}$ island hypermethylation, but $p 53$ and KRAS mutations are not. In fact, $p 53$ mutations distinguish the cancer as evolving outside of the $\mathrm{CpG}$ island hypermethylation genotype. Also, CpG island hypermethylation-associated colon cancers often occur in the right colon. ${ }^{1,3}$

\section{KRAS and BRAF Testing in CRC}

From a treatment perspective, targeted therapy is available for patients with rectal cancer. Somatic mutations (mutations occurring in the tumor DNA) in KRAS are known to confer resistance to anti-EGFR therapy such as cetuximab and panitumumab. ${ }^{4-6}$ The National Cancer Comprehensive Network (NCCN) guidelines recommend "expanded" RAS testing to include both KRAS and NRAS codons 12, 13 and 61 (and codon 146 in KRAS) in metastatic cases. ${ }^{4}$ BRAF testing, specifically for V600E mutations, is known to have prognostic implications, and, at the time of this paper, trials with drugs that target $B R A F$ are in the initial phases. Other potentially clinically relevant targets in the future include PIK3CA and PTEN. ${ }^{5,6}$ Additional genomic considerations regarding therapy that are not currently tested universally include pharmacogenetic considerations of drug therapy such as UGT1A $1 * 28$ mutations and irinotecan toxicity, and DYPD mutations for 5fluorouracil or capecitabine toxicity. All of these mutations are germline in origin (present in every nucleated cell in the body, detected through blood or saliva samples). ${ }^{7,8}$ Lastly, determination of the presence or absence of a hereditary cancer syndrome (expanded in the subsequent sections) is important because individuals with Lynch Syndrome who are treated with 5-fluoruouracil are documented to have no improvement; or, in some cases have worse outcomes. ${ }^{9}$

\section{Hereditary Cancer Syndromes}

Multiple syndromes exist with a predisposition to cancer that involves the rectum. The comprehensive list includes familial adenomatous polyposis coli (FAP), Lynch syndrome, PeutzJeghers syndrome (PJS), phosphatase and tensin homolog (PTEN) hamartomatous syndrome, juvenile polyposis syndrome, $M Y H$-associated polyposis, hereditary mixed polyposis, serrated polyposis, and POLE/POLD1. With the exception of one, each of these syndromes is inherited in an autosomal dominant fashion. The exception to this is $\mathrm{MYH}$-associated polyposis, which is autosomal recessive. It is important to consider the inheritance pattern when trying to establish the patient's genetic risk, as well as risk to the patient's relatives. Moreover, comprehensive knowledge of the features of each type of hereditary rectal cancer is important when developing the family history, since a syndrome, by definition, is a "constellation of symptoms," to avoid overlooking an individual's hereditary predilection for cancer. Each syndrome will be considered individually herein.

\section{Familial Adenomatous Polyposis Coli}

FAP was first described in $1859 .{ }^{10}$ This disorder accounts for $\leq 0.5 \%$ of colorectal carcinoma and is classically characterized by hundreds to thousands of adenomas that are present throughout the length of the large bowel and rectum. ${ }^{11-14}$ Polyps usually present in the second decade of life, and an individual with FAP's lifetime risk of colorectal carcinoma is $100 \%$, with a mean age at diagnosis of 36 years.

Inherited in an autosomal dominant fashion, affected individuals have a $50 \%$ chance of passing the mutated allele 


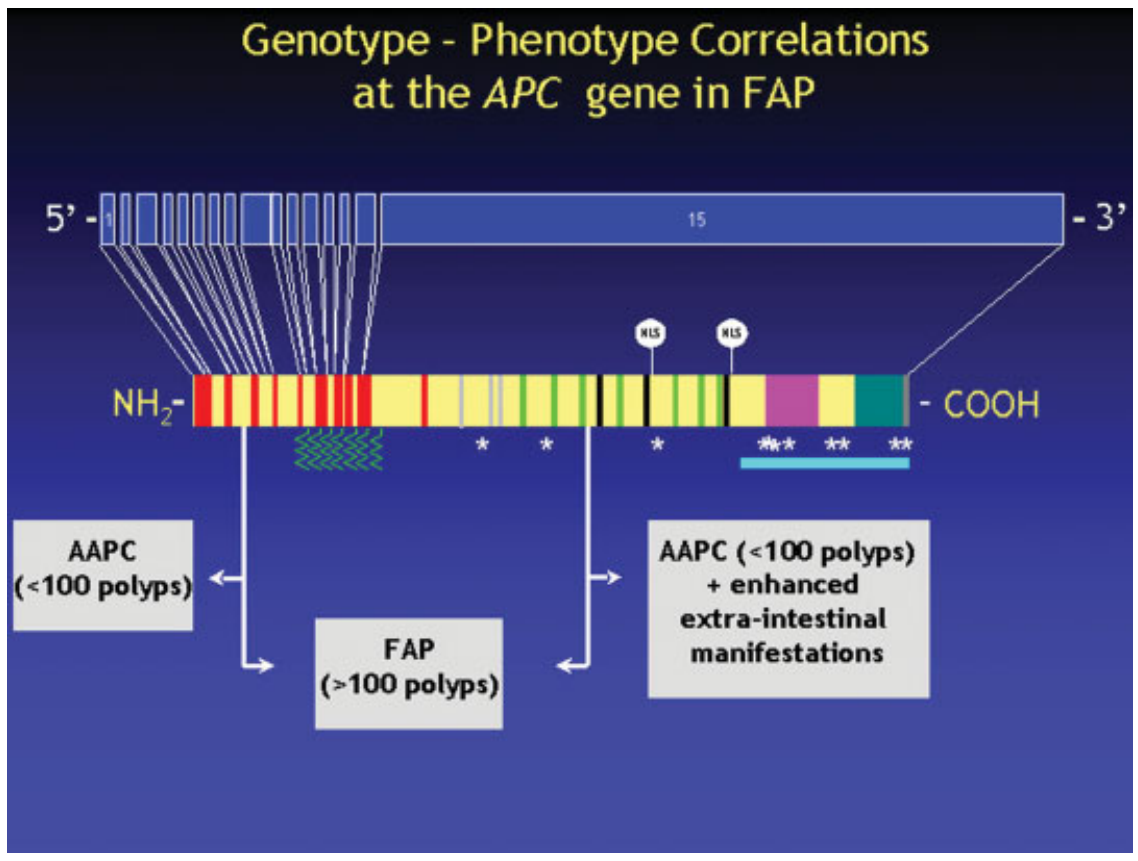

Fig. 2 Genotype-phenotype correlations of FAP. FAP, familial adenomatous polyposis coli.

to each offspring, and all who inherit the mutation will manifest the disease (complete penetrance). The gene involved is the APC tumor-suppressor gene, located on $5 \mathrm{q} 21$, and, as is the case with all hereditary disorders, the mutation is present in every nucleated cell within the body. The diagnosis is confirmed by sequencing the gene on a blood sample from an affected individual. While an inherited disorder, a high de novo mutation rate exists (as high as $25 \%$ ), and greater than $95 \%$ of mutations result in a truncated protein. ${ }^{14}$ De novo mutation indicates that this is the first time the mutation is seen within the family, and that it occurred early in the embryologic development, but as a postzygotic event. Small insertions or deletions are the most common mutations that either result in a shift in the reading frame leading to a premature stop codon, hence a "truncated protein," or confer a premature stop codon by the nature of the change in bases altogether. Deletions and duplications and splice site changes also occur. ${ }^{12,14}$

Although the most devastating result of FAP is colorectal cancer, extraintestinal manifestations exist. Congenital hyperpigmentation of the retinal epithelium is one of these features, as well as fundic gland polyps in the stomach, and duodenal polyps. Extraintestinal malignancies are also seen involving the duodenum and the brain. Medulloblastoma is the brain malignancy associated with FAP, and the concomitant presence of FAP and medulloblastoma is known as Turcot syndrome. The presence of desmoids, osteomas (especially in the face or jaw), dental abnormalities, and epidermal cysts is Gardner syndrome. Papillary thyroid carcinoma, periampullary adenocarcinoma, pancreatic adenocarcinoma, hepatoblastoma, and gastric adenocarcinoma are additional extracolonic malignancies seen in FAP. ${ }^{12,14}$

FAP is a classic example of a disorder with genotypephenotype correlations which means that mutations occur- ring in particular regions of the gene confer a specific phenotype (-Fig. 2). Specifically, mutations occurring toward the $5^{\prime}$ end of the gene, involving exons $1-9$, and the extreme $3^{\prime}$ end are associated with fewer than 100 polyps, and therefore individuals with this presentation are diagnosed with attenuated FAP. In addition, individuals with attenuated FAP tend to have right-sided carcinoma, and present with colorectal carcinoma later in life (56 years of age, on average) than individuals with the other forms of FAP. Mutations occurring in the middle portion of the gene, including exons 5 through the 5 ' end of exon 15 are associated with manifestations of classic FAP. Severe polyposis is associated with codons 1250-1464, and desmoids tumors with mutations in codons 1445-1578, both of which exist in codon 15 , upstream from the second mutation hotspot associated with an attenuated FAP phenotype. ${ }^{12,14}$

In patients with classic FAP, the risk of developing carcinomatous change in the colon and rectum is $100 \%$ if left untreated. Similarly, patients with FAP who undergo ileorectal anastomosis (IRA) are at risk of developing a rectal cancer, with reports of the development of carcinoma in up to $29 \% .{ }^{15-17}$ Furthermore, the survival rate of patients with FAP who develop a rectal cancer following IRA is significantly reduced. ${ }^{18,19}$ Therefore, restorative prophylactic proctocolectomy with ileoanal pouch anal anastomosis (IPAA) is the surgical treatment of choice offered to patients with FAP.

Although less controversial now, handsewn with mucosectomy versus stapled IPAA continues to be debated. Stapled IPAA is associated with improved functional outcomes with the primary concern being the rectal mucosa remaining along the transitional zone. However, the actual risk of cancer in the anal transition zone is quite low with only case reports described. $^{20-27}$ In contrast, mucosectomy and handsewn technique removes this residual rectal mucosa. However, 
islets of primary rectal mucosa have been reported in up to $20 \%$ of pouches excised, thus allowing for the potential of malignancy. ${ }^{28}$

In patients with attenuated FAP, rectal sparing may allow for sparing of the rectum and creation of an IRA. However, the rectum must be free of polyps and the patient must be compliant with intense screening of the remaining rectum.

\section{Lynch Syndrome}

Lynch syndrome, also historically referred to as hereditary nonpolyposis colorectal cancer syndrome, occurs in approximately 2 to $3 \%$ of colorectal carcinomas, with a prevalence of $1: 440$ in the population. Early description of clustering of malignancies ultimately identified as Lynch syndrome began in 1895 with observations by Aldred Scott Warthin, MD, PhD, at the University of Michigan in Ann Arbor. The family Dr. Warthin described emigrated from Germany to Michigan before the Civil War. The family included his patient with a sibship comprised 10 members with the following cancers: two uterine, two stomach, and one "abdominal" cancer. All five of these siblings with a personal history of cancer had descendants with multiple cancers, demonstrating Lynch syndrome's autosomal dominant pattern of inheritance. Moreover, the offspring of the five siblings without cancer also did not develop cancer. This family was entitled "Family G" (for their German origin). Some time passed with intermittent descriptions of and interest in familial colorectal carcinoma other than FAP, which was ultimately embraced by Henry T. Lynch, MD, for whom the eponym derives it designation, so betrothed by $\mathrm{C}$. Richard Boland, MD, PhD. Specifically, the term Lynch syndrome implies colorectal carcinoma or associated extraintestinal manifestations in families with a germline mutation in DNA MMR genes. More research was conducted, and ultimately, in 1993, nearly 100 years following its first description of familial gastrointestinal cancers, MSI was discovered by independent groups including Thibodeau at the Mayo Clinic, Rochester, and Vogelstein at Johns Hopkins and international colleagues from Finland de la Chapelle, Aaltonen, and Peltomaki. This began a rush to discover the genes involved in producing these microsatellites, which were already understood to be part of the MMR system in DNA. Although several MMR proteins have been described, including MLH1, MLH3, PMS1, PMS2, MSH2, MSH3, MSH6, TGFßR2, and EXO1, the main contributors to the development of Lynch syndrome are MLH1, PMS2, MSH2, and MSH6 (-Fig. 3). The proteins function as heterodimers, MLH1 coupled with PMS2, and MSH2 coupled with MSH6. ${ }^{3,29,30}$

As the data materialized, diagnostic guidelines were developed, the first of which was the Amsterdam Criteria. Following this, the Amsterdam Criteria was improved upon in Bethesda, MD, in 1997, with establishment of the "Be-

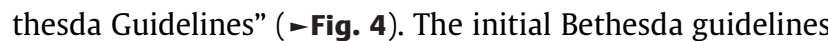
were revised in 2004 with the realization that the established guidelines were missing many individuals with Lynch syndrome. Still yet, the second revisions were not comprehensive enough, leading to a consensus following a workshop in Jerusalem in 2009 that any individual younger than 70 years

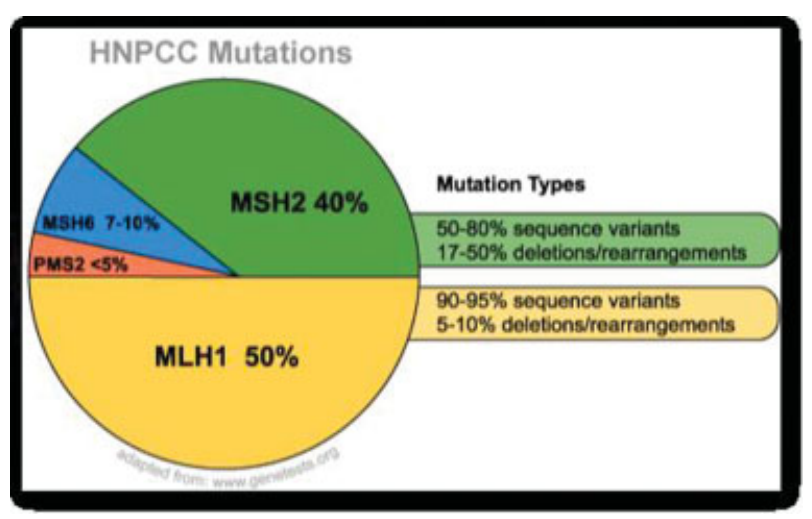

Fig. 3 MMR proteins involved in Lynch syndrome.

who presents with colorectal carcinoma should be screened with either IHC for assessment of the expression of MMR proteins or with polymerase chain reaction (PCR) for MSI, both of which are conducted on tumor tissue. Each technique has advantages and disadvantages with, overall slightly greater sensitivity with MSI. Another challenge of IHC is the possibility of discordant results due to the mutation not completely eliminating the ability of the protein to be expressed, yet MSI detected in the tumor. ${ }^{29}$ With the advantage of time, genotype-phenotype correlations are also observed in Lynch syndrome. For example, PMS2 (7p22.1) is rarely involved. This gene is flanked by a pseudogene which makes the testing very complicated. As such, while the present understanding is that mutations in PMS2 are less common in Lynch syndrome, techniques to sequence this gene have been successfully developed in the past few years, which may change the frequency of PMS2 mutations, and hence its

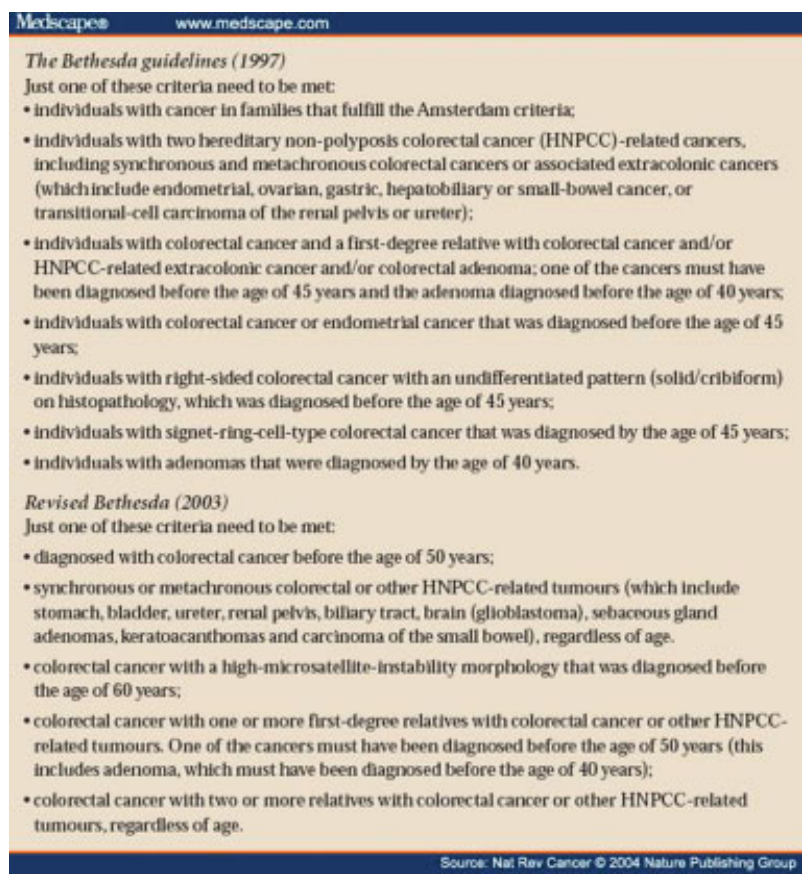

Fig. 4 Bethesda guidelines. 
prevalence as a contributor to Lynch syndrome. MSH6 (2p16.3) has an increased incidence of uterine cancer and more distally located colon cancers. MSH6 is also associated with the finding of MSI-L in MSI studies. Mutations in MSH2 (2p21) are associated with the greatest risk of extracolonic cancers, especially when an upstream promoter, EPCAM is deleted. However, a lower risk of endometrial cancer is associated with EPCAM deletions. Moreover, $M S H 2$ mutations are often seen in Muir-Torre syndrome. Of the four genes, MLH1 (3p22.2) and MSH2 are the most commonly involved in the colon and rectum. ${ }^{13,29,30}$

Lynch syndrome-related colorectal carcinomas have a particular morphology including a poorly differentiated lesion with tumor-infiltrating lymphocytes, mucinous differentiation, signet ring cells, a pushing margin, and a Crohn-like reaction. Carcinomas tend to be located in the right colon. Moreover, these malignancies usually present at low stage. ${ }^{31-33}$

Determination of a colorectal carcinoma as a Lynch syndrome malignancy is essential as it will influence management of the malignancy not only with respect to surgical consideration but also with chemotherapeutic regimens and surveillance follow-up. Specifically individuals with Lynch syndrome who received 5-fluorescence have a worse outcome. The known extraintestinal manifestations of Lynch syndrome include endometrial endometrioid adenocarcinoma (most commonly, occasionally clear cell, papillary serous carcinoma, malignant mixed Müllerian tumor, and less frequently other histologic patterns), ovarian endometrioid adenocarcinoma, small bowel adenocarcinoma, gastric adenocarcinoma, pancreatic adenocarcinoma, biliary carcinoma, carcinoma of the ureter, bladder, and renal pelvis, sebaceous gland tumors (Muir-Torre syndrome), keratoacanthomas, and glioblastoma multiforme (Turcot syndrome). The overall cancer risks are as follows: colorectal carcinoma 52 to $82 \%$ (mean age at diagnosis 44-61 years), endometrial carcinoma 25 to $60 \%$ (mean age at diagnosis 48-62 years), gastric cancer 6 to $13 \%$ (mean age at diagnosis 56 years), ovarian carcinoma 4 to $12 \%$ (usually diagnosed by the age of 40 years), and much lower risk for the other extraintestinal malignancies. ${ }^{13,29}$

Surgeons may be the initial physician to discover a pattern consistent with Lynch syndrome, thus it is imperative for surgeons to understand the associated malignancies as well as the implications of the disease as it relates to surgery. As mentioned, the most common cancer associated with Lynch syndrome is colorectal origin with the risk of endometrial cancer approximately equal to that of colorectal. Furthermore, there is a reported approximate risk of $40 \%$ at 10 years from diagnosis in which patients with Lynch syndrome are at risk of developing metachronous colorectal cancers. ${ }^{34,35} \mathrm{Al}-$ though the overall function in patients with segmental resection has shown to be better, the risk of metachronous cancer typically outweighs the benefit of function. Thus, most surgeons perform an extended resection consisting of a subtotal colectomy with IRA if the rectum is spared of disease as opposed to a segmental resection as is standard for a sporadic cancer. Similarly, given the increased incidence of endometrial cancer, prophylactic hysterectomy is warranted, which may be done synchronously.
The estimated risk of rectal cancer in patients with Lynch syndrome has been reported in the surgical literature to be as high as $20 \%$. Thus, patients with Lynch syndrome and a diagnosis of rectal cancer should be evaluated preoperatively in a similar manner to patients with sporadic rectal cancer. ${ }^{35-38}$ Thus, if the staging meets criteria for neoadjuvant therapy, the patient should receive appropriate therapy. The operation offered, however, may differ in that a total proctocolectomy may be offered. In the absence of neoadjuvant chemotherapy and radiation, a total proctocolectomy with ileoanal pouch anastomosis may be constructed. The ileoanal pouch remains at risk of neoplastic change and requires surveillance regularly.

\section{MUTYH Polyposis}

As mentioned earlier, MUTYH polyposis (MAP) is inherited in an autosomal recessive fashion and therefore requires two mutations (biallelic) to manifest the disease. The MUTYH gene is a base excision repair gene, and MUTYH polyposis is characterized by multiple adenomas ( $>10$ on the average) and absence of a mutation in the APC gene (cases without polyps are also seen). These patients may present at a later age with polyps distributed along the entire length of the colon, but do not exclude the small bowel, especially the duodenum. In addition, the polyps may be the usual tubular adenomas but can also include hyperplastic polyps, serrated adenomas, and sessile serrated adenomas. ${ }^{39}$ Clinically, the phenotype can parallel Lynch syndrome, including other extracolonic manifestations, and it is documented that clinical recognition of this entity is under-ascertained, with a study of 405 colorectal carcinomas in Spain containing an incidence of 27 (7\% incidence) confirmed cases with MUTYH polyposis. ${ }^{39-42}$ The phenotypic similarity to Lynch syndrome has included sebaceous lesions, and loss of MMR protein expression histologically (including MSH2 and MSH6), with tumor MSI. ${ }^{40}$ Other associated extracolonic malignancies involve the breast, thyroid, testis, and hematologic. ${ }^{41}$ Testing for KRAS was positive $67 \%$ of the time in individuals with confirmed biallelic MUTYH mutations, suggesting value of KRAS testing before germline testing when the question of MUTYH polyposis is present clinically. Specifically, the c.34G > T, p.G12C is the somatic transversion identified most often, and routine KRAS tests would cover the codons that have been documented thus far in KRAS in individuals with MUTYH polyposis. ${ }^{43}$ When an individual is known to be affected, colonoscopy beginning at the age of 20 years is recommended. However, the autosomal recessive inheritance pattern precludes early detection, and most individuals present with invasive cancer. ${ }^{39,43}$

\section{Juvenile Polyposis Syndrome}

Juvenile polyposis syndrome (JPS) is an autosomal dominant condition with a frequency between $1: 16,000$ and $1: 100,000$ in which the individual is predisposed to hamartomatous polyps throughout the gastrointestinal tract beginning before the age of 20 years with an increased risk of carcinoma that varies between 9 and 50\%. ${ }^{11,44-46}$ Nearly $75 \%$ of 
individuals have an affected parent, de novo mutations occur in $25 \%$ of cases. The predominant cancer risk exists in the colon, but cases of gastric, upper gastrointestinal tract, and pancreas cancer have been documented. It is important to recall that some individuals with a mutation in SMAD4, one of three genes associated with this disorder, may also develop hereditary hemorrhagic telangiectasia (HHT) 15 to $22 \%$ of the time, which is subsequently referred to as HHT/JPS. Individuals with JPS who harbor SMAD4 mutations have a greater proportion of gastric polyposis and an increased risk for gastric cancer. ${ }^{45}$ The other identified genes are BRMP1A (10q22q23) and ENG (9Q34.11), all of which are associated with transforming-growth factor- $\beta$. Collectively, each gene accounts for the genetic diagnosis of JPS 50 to $60 \%$ of the time. ${ }^{44}$

Although there is variability, most individuals have at least four to five polyps, presenting early in life. Some individuals will develop many polyps. Morphologically, the hamartomatous polyp of JPS is unique from that associated with Cowden syndrome (CS). Although the polyps in CS are characterized by streams of smooth muscle extending in an arborizing pattern, JPS hamartomatous polyps are characterized by a normal epithelium with a dense stroma containing an inflammatory infiltrate, mucus-filled cystically dilated glands in the lamina propria, and a smooth surface, sometimes with neoplastic changes. ${ }^{11,44,46}$ Additional clinical features of JPS include midgut malrotation, heart defects, cleft palate, cranial abnormalities, and polydactyly. ${ }^{45}$

\section{Peutz-Jeghers Syndrome}

Clinically, PJS presents with mucocutaneous melanosis and hamartomatous polyps. Again, the inheritance is autosomal dominant, and it is the second most common form of intestinal polyposis, with an incidence 1/10 that of FAP. The gene responsible for up to $70 \%$ of cases is a tumor suppressor gene, STK11 (LKB1), a serine threonine kinase receptor with a role in cell cycle regulation, regulation of apoptosis, and with cell cycle regulation. Penetrance is variable and incomplete. The diagnosis can be made in infancy, with macules developing within the first 2 years of life. However, the average age of diagnosis is 25 years. Small bowel lesions are often symptomatic, aiding in the diagnosis. There is a 39\% increased risk of colorectal carcinoma in individuals with PJS, as well as an increased risk of malignancy in extraintestinal sites including: the breast (second most common malignancy), uterine cervix, testis, pancreas, and ovary. ${ }^{11,46,47}$ With respect to the uterine cervix, adenoma malignum is one diagnosis that increases the clinical suspicion of PJS. The mean age of cancer diagnosis is 43 years. The hamartomatous polyps characteristic of PJS are located throughout the gastrointestinal tract, with a predilection for the ileum, jejunum, and colon. ${ }^{11,46,47}$ Histologically, these polyps contain fronds of smooth muscle in an arborizing pattern, which is seen more easily in the polyps that are located in the small bowel. ${ }^{47}$

\section{PTEN Hamartomatous Syndrome}

As the name implies, PTEN hamartomatous syndrome is associated with mutations in the PTEN tumor suppressor gene and includes both CS and Bannayan Riley Ruvalcaba Syndrome (BRRS). The pattern of inheritance is autosomal dominant. While breast, thyroid, and endometrial malignancies are associated with CS, rectal carcinoma is also known to occur. Again, hamartomatous lesions are present in the gastrointestinal tract of individuals with CS, as well as BRRS. Physical examination distinguishes these two entities, since macrocephaly and polyps are seen with each. In BRRS, pigmented macules are present on the penis. Dysplastic gangliocytomas of the cerebellum are said to be pathognomonic for CS, a diagnosis otherwise known as Lhermitte-Duclos. The polyps seen in PTEN hamartomatous syndromes can include hamartomas (most commonly), adenomas, ganglioneuromas, inflammatory polyps, and less commonly leiomyomas, lipomatous, and lymphoid polyps. Polyps have varied from few to more than hundreds in quantity, with the potential for the presence of more than one histologic type present synchronously. The colorectal cancer risk has been stated to vary from 9 to $16 \%$ lifetime risk and is thought to emerge likely from a preexisting adenomatous polyp, or a hamartoma. ${ }^{11,46,47} \mathrm{Ge}-$ netic confirmation of the diagnosis of CS occurs in $80 \%$ of cases when the diagnostic criteria is applied accurately. ${ }^{47}$ Extraintestinal manifestations of CS include trichilemmomas and acral keratoses, and an increased risk of breast carcinoma, thyroid carcinoma (papillary thyroid most often), as well as renal cell carcinoma (several histologic variants), melanoma, and endometrial carcinoma (the latter two malignancies seen less often). ${ }^{47-49}$

Other syndromes associated with hamartomatous polyps include Gorlin syndrome (PTCH1), multiple endocrine neoplasia 2B (RET), neurofibromatosis type 1 (NF1), and BirtHogg Dube $(F L C N){ }^{47}$

\section{Hereditary Mixed Polyposis}

Inherited in an autosomal dominant fashion, this condition is characterized by a presentation with a variety of polyps including tubular adenomas, juvenile polyps, and hyperplastic polyps. A few genes have been identified as candidates that contribute to this syndrome, and additional details regarding extracolonic manifestations are not as well established as the previously discussed entities, as it is one of the newer polyposis syndromes described. Candidate genes identified thus far include: BMPR1A (note also seen in juvenile polyposis), GREM1, and SCG5. ${ }^{11,46,50,51}$

\section{Serrated Polyposis}

This entity remains genetically elusive but is diagnosed in accordance with the World Health Organization criteria published by Snover et al in 2010. An increased risk of CRC is present in patients with serrated polyposis, with an autosomal dominant inheritance pattern. Tubular adenomas can also be present in individuals with serrated polyposis, and, to date, two genes have been described with a potential role in this syndrome: PTEN, MUTYH. ${ }^{11,41}$ However, other than the two mentioned genes, genome-wide association studies have demonstrated considerable genetic heterogeneity in individuals with serrated polyposis, making targeted testing difficult. ${ }^{52}$ 


\section{Polymerase Proofreading Polyposis}

New genes, POLE (polymerase $\varepsilon$ ) and POLD1 (polymerase $\delta$ ), the equivalent lagging strand polymerase to POLE, have been identified as candidate hereditary colorectal carcinoma genes, with affected individuals presenting with 10 to 100 polyps, microsatellite stable tumors, under the age of 60 years, and an autosomal dominant pattern of inheritance. In many cases, a family history of colorectal carcinoma is present, though this is not always the case. At the time of completion of this article, these genes are just identified, and additional data regarding their status are needed. ${ }^{53}$

\section{Summary}

In summary, the majority of rectal carcinomas are sporadic in nature, and relevant testing for driver mutations to guide therapy is important. However, a thorough family history is necessary and helpful in elucidating a potential hereditary predilection for the patient's carcinoma. Moreover, adequate diagnosis of a heritable tendency toward CRC will alter the management of the patient's known disease, in some instances, and permit the implementation of various surveillance algorithms as a preventive measure.

\section{References}

1 Bhalla A, Zulfiqar M, Weindel M, Shidham VB. Molecular diagnostics in colorectal carcinoma. Clin Lab Med 2013;33(4): 835-859

2 Brenner H, Kloor M, Pox CP. Colorectal cancer. Lancet 2014; 383(9927):1490-1502

3 Boland CR, Goel A. Microsatellite instability in colorectal cancer. Gastroenterology 2010;138(6):2073-2087.e3

4 Kircher SM, Mohindra N, Nimeiri H. "http://www.ncbi.nlm.nih. gov/pubmed/25410095" Cost estimates and economic implications of expanded RAS testing in metastatic colorectal cancer. Oncologist 2015;20(1):14-18

5 Wendy DeRoock, Bart Claes, David Bernasconi, et al. Effects of KRAS, BRAF, NRAS, and PIK3CA mutations on the efficacy of cetuximab plus chemotherapy in chemotherapy-refractory metastatic colorectal cancer: a retrospective consortium analysis. Lancet Oncol 2010;11(8):753-762

6 Wendy DeRoock, Veerle DeVriendt, Nicola Normanno, Fortunato Ciardiello, Sabine Tejpar. KRAS, BRAF, PIK3CA, and PTEN mutations: implications for targeted therapies in metastatic colorectal cancer. Lancet Oncol 2011;12(6):594-603

7 Tanja K. Froehlich, Ursula Amstutz, Stefan Aebi, Markus Joerger, Carlo R. Largiadèr. Clinical importance of risk variants in the dihydropyrimidine dehydrogenase gene for the prediction of early-onset fluoropyrimidine toxicity. Int J Cancer 2014;136(3): 730-739

8 Inoue K, Sonobe M, Kawamura Y, et al. Polymorphisms of the UDPglucuronosyl transferase $1 \mathrm{~A}$ Genes are associated with adverse events in cancer patients receiving irinotecan-based chemotherapy. Tohoku J Exp Med 2013;229:107-114

9 Buecher B, Cacheux W, Rouleau E, et al. Role of microsatellite instability in the management of colorectal cancers. Dig Liver Dis 2013;45:441-449

10 Chargelaigue A. Des Polypes du Rectum. Thesis Paris; 1859

11 Lucci-Cordisco E, Risio M, Venesio T, Genuardi M. The growing complexity of the intestinal polyposis syndromes. Am J Med Genet A 2013;161A(11):2777-2787
12 Sieber OM, Tomlinson IP, Lamlum H. The adenomatous polyposis coli (APC) tumour suppressor-genetics, function and disease. Mol Med Today 2000;6(12):462-469

13 Lindor NM. Recognition of genetic syndromes in families with suspected hereditary colon cancer syndromes. Clin Gastroenterol Hepatol 2004;2(5):366-375

14 Kerr SE, Thomas CB, Thibodeau SN, Ferber MJ, Halling KC. APC germline mutations in individuals being evaluated for familial adenomatous polyposis: a review of the Mayo Clinic experience with 1591 consecutive tests. J Mol Diagn 2013;15(1):31-43

15 Church J, Burke C, McGannon E, Pastean O, Clark B. Risk of rectal cancer in patients after colectomy and ileorectal anastomosis for familial adenomatous polyposis: a function of available surgical options. Dis Colon Rectum 2003;46(9):1175-1181

16 Vasen HF, van Duijvendijk P, Buskens E, et al. Decision analysis in the surgical treatment of patients with familial adenomatous polyposis: a Dutch-Scandinavian collaborative study including 659 patients. Gut 2001;49(2):231-235

17 Nugent KP, Phillips RK. Rectal cancer risk in older patients with familial adenomatous polyposis and an ileorectal anastomosis: a cause for concern. Br J Surg 1992;79(11):1204-1206

18 Bertario L, Presciuttini S, Sala P, Rossetti C, Pietroiusti M; Italian Registry of Familial Polyposis Writing Committee. Causes of death and postsurgical survival in familial adenomatous polyposis: results from the Italian Registry. Semin Surg Oncol 1994;10(3):225-234

19 De Cosse JJ, Bülow S, Neale K, et al; The Leeds Castle Polyposis Group. Rectal cancer risk in patients treated for familial adenomatous polyposis. Br J Surg 1992;79(12):1372-1375

20 Tuckson W, Lavery I, Fazio V, Oakley J, Church J, Milsom J. Manometric and functional comparison of ileal pouch anal anastomosis with and without anal manipulation. Am J Surg 1991; 161(1):90-95, discussion 95-96

21 Church JM, Oakley JR, Wu JS. Pouch polyposis after ileal pouch-anal anastomosis for familial adenomatous polyposis: report of a case. Dis Colon Rectum 1996;39(5):584-586

22 Remzi FH, Church JM, Bast J, et al. Mucosectomy vs. stapled ileal pouch-anal anastomosis in patients with familial adenomatous polyposis: functional outcome and neoplasia control. Dis Colon Rectum 2001;44(11):1590-1596

23 van Duijvendijk P, Vasen HF, Bertario L, et al. Cumulative risk of developing polyps or malignancy at the ileal pouch-anal anastomosis in patients with familial adenomatous polyposis. J Gastrointest Surg 1999;3(3):325-330

24 Ziv Y, Fazio VW, Sirimarco MT, Lavery IC, Goldblum JR, Petras RE. Incidence, risk factors, and treatment of dysplasia in the anal transitional zone after ileal pouch-anal anastomosis. Dis Colon Rectum 1994;37(12):1281-1285

25 Remzi FH, Fazio VW, Delaney CP, et al. Dysplasia of the anal transitional zone after ileal pouch-anal anastomosis: results of prospective evaluation after a minimum of ten years. Dis Colon Rectum 2003;46(1):6-13

26 Ooi BS, Remzi FH, Gramlich T, Church JM, Preen M, Fazio VW. Anal transitional zone cancer after restorative proctocolectomy and ileoanal anastomosis in familial adenomatous polyposis: report of two cases. Dis Colon Rectum 2003;46(10):1418-1423, discussion 1422-1423

27 Vrouenraets BC, Van Duijvendijk P, Bemelman WA, Offerhaus GJ, Slors JF. Adenocarcinoma in the anal canal after ileal pouch-anal anastomosis for familial adenomatous polyposis using a doublestapled technique: report of two cases. Dis Colon Rectum 2004; 47(4):530-534

28 O'Connell PR, Williams NS. Mucosectomy in restorative proctocolectomy. Br J Surg 1991;78(2):129-130

29 Lynch HT, Lynch PM, Lanspa SJ, Snyder CL, Lynch JF, Boland CR. Review of the Lynch syndrome: history, molecular genetics, screening, differential diagnosis, and medicolegal ramifications. Clin Genet 2009;76(1):1-18 
30 Jasperson KW, Tuohy TM, Neklason DW, Burt RW. Hereditary and familial colon cancer. Gastroenterology 2010;138(6):2044-2058

31 Smyrk TC, Watson P, Kaul K, Lynch HT. Tumor-infiltrating lymphocytes are a marker for microsatellite instability in colorectal carcinoma. Cancer 2001;91(12):2417-2422

32 Alexander J, Watanabe T, Wu TT, Rashid A, Li S, Hamilton SR. Histopathological identification of colon cancer with microsatellite instability. Am J Pathol 2001;158(2):527-535

33 Jass JR. Pathology of hereditary nonpolyposis colorectal cancer. Ann N Y Acad Sci 2000;910:62-73, discussion 73-74

34 Fitzgibbons RJ Jr, Lynch HT, Salerno GM. Hereditary colon cancer syndromes. Surg Annu 1991;23(Pt 2):111-132

35 Parry S, Win AK, Parry B, et al. Metachronous colorectal cancer risk for mismatch repair gene mutation carriers: the advantage of more extensive colon surgery. Gut 2011;60(7):950-957

36 Natarajan N, Watson P, Silva-Lopez E, Lynch HT. Comparison of extended colectomy and limited resection in patients with Lynch syndrome. Dis Colon Rectum 2010;53(1):77-82

37 You YN, Chua HK, Nelson H, Hassan I, Barnes SA, Harrington J. Segmental vs. extended colectomy: measurable differences in morbidity, function, and quality of life. Dis Colon Rectum 2008; 51(7):1036-1043

38 Cirillo L, Urso ED, Parrinello G, et al. High risk of rectal cancer and of metachronous colorectal cancer in probands of families fulfilling the Amsterdam criteria. Ann Surg 2013;257(5):900-904

39 Borras E, Taggart MW, Lynch PM, Vilar E. Establishing a diagnostic road map for MUTYH-associated polyposis. Clin Cancer Res 2014; 20(5):1061-1063

40 Morak M, Heidenreich B, Keller G, et al. Biallelic MUTYH mutations can mimic Lynch syndrome. Eur J Hum Genet 2014;22(11): 1334-1337

41 Guarinos C, Sánchez-Fortún C, Rodríguez-Soler M, et al. Clinical subtypes and molecular characteristics of serrated polyposis syndrome. Clin Gastroenterol Hepatol 2013;11(6):705-711, quiz e46

42 Landon M, Ceulemans S, Saraiya DS, et al. Analysis of current testing practices for biallelic MUTYH mutations in MUTYH-associated polyposis. Clin Genet 2014; •*: 10.1111/cge.12375
43 Castillejo A, Vargas G, Castillejo MI, et al. Prevalence of germline MUTYH mutations among Lynch-like syndrome patients. Eur J Cancer 2014;50(13):2241-2250

44 Brosens LA, Langeveld D, van Hattem WA, Giardiello FM, Offerhaus GJ. Juvenile polyposis syndrome. World J Gastroenterol 2011; 17(44):4839-4844

45 Latchford AR, Neale K, Phillips RK, Clark SK. Juvenile polyposis syndrome: a study of genotype, phenotype, and long-term outcome. Dis Colon Rectum 2012;55(10):1038-1043

46 Patel SG, Ahnen DJ. Familial colon cancer syndromes: an update of a rapidly evolving field. Curr Gastroenterol Rep 2012;14(5): 428-438

47 Jelsig AM, Qvist N, Brusgaard K, Nielsen CB, Hansen TP, Ousager LB. Hamartomatous polyposis syndromes: a review. Orphanet J Rare Dis 2014;9:101

48 Pilarski R, Burt R, Kohlman W, Pho L, Shannon KM, Swisher E. Cowden syndrome and the PTEN hamartoma tumor syndrome: systematic review and revised diagnostic criteria. J Natl Cancer Inst 2013;105(21):1607-1616

49 Shuch B, Ricketts CJ, Vocke CD, et al. Germline PTEN mutation Cowden syndrome: an underappreciated form of hereditary kidney cancer. J Urol 2013;190(6):1990-1998

50 Jaeger E, Leedham S, Lewis A, et al. Hereditary mixed polyposis syndrome is caused by a $40-\mathrm{kb}$ upstream duplication that leads to increased and ectopic expression of the BMP antagonist GREM1. Nat Genet 2012;44(6):699-703

51 Whitelaw SC, Murday VA, Tomlinson IP, et al. Clinical and molecular features of the hereditary mixed polyposis syndrome. Gastroenterology 1997;112(2):327-334

52 Gala MK, Mizukami Y, Le LP, et al. Germline mutations in oncogene-induced senescence pathways are associated with multiple sessile serrated adenomas. Gastroenterology 2014; 146(2):520-529

53 Palles C, Cazier JB, Howarth KM, et al; CORGI Consortium; WGS500 Consortium. Germline mutations affecting the proofreading domains of POLE and POLD1 predispose to colorectal adenomas and carcinomas. Nat Genet 2013;45(2):136-144 